\title{
Modal and Stress Analysis of Cellular Structures Produced with Additive Manufacturing by Finite Element Analysis (FEA)
}

\author{
${ }^{1}$ Bekir Yalçın, *2'Berkay Ergene, ${ }^{3}$ Uçan Karakılınç \\ ${ }^{1}$ Faculty of Technology, Department of Mechanical and Manufacturing Engineering, Isparta Applied Sciences \\ University, Isparta, Turkey \\ ${ }^{* 2}$ Faculty of Technology, Department of Mechanical and Manufacturing Engineering, Isparta Applied Sciences \\ University, Isparta, Turkey \\ ${ }^{3}$ YETEM Research and Practice Center, Design and Manufacturing Department, Isparta, Turkey
}

\begin{abstract}
Cellular structures such as regular/irregular honeycombs and re-entrants are known as lighter, high level flexibility and more efficient materials; these cellular structures have been mainly designed with topology optimization and obtained with new additive manufacturing methods for aircraft industry, automotive, medical, sports and leisure sectors. For this aim, the effect of cellular structures such as the honeycomb and re-entrant on vibration and stress-strain behaviors were determined under compression and vibration condition by finite elements analyses (FEA). In FEA, the re-entrant and honeycomb structures were modeled firstly and then the stress and displacement values for each structure were obtained. Secondly, vibration behaviors of these foam structures were estimated under determined boundary conditions. In conclusion, the effect of topology in foam structures on vibration and mechanical behaviour were exhibited in FEA results. The obtained stress results of FEA show that all stresses $\left(\sigma_{\mathrm{x}}, \sigma_{\mathrm{y}}, \sigma_{\mathrm{vm}}, \tau_{\mathrm{xy}}\right)$ are lower on honeycomb structure than reentrant structure. Besides, natural frequency values $\left(\omega_{1}, \omega_{2}, \omega_{3}\right)$ and appearance of each structure were observed by using FEA.
\end{abstract}

Key words: Cellular structure, honeycomb, re-entrant, finite element analysis, vibration.

\section{Introduction}

The cellular (foam) structures are increasingly more important due to their specific properties such as lightest, thermal insulation, good damping capacity at low stresses and more flexibility. The cellular material mechanic is different from conventional engineering material. With this context, the engineering materials are characterized by a positive Poisson's ratio according to classic elasticity theory, they are observed to shrink (expand) laterally when stretched (compressed) longitudinally. Unlike the classical theory of elasticity, new one of the cellular structures is known as "auxetic" has a negative Poisson' ratio, they show behaviour to expand (shrink) laterally when stretched (compressed) longitudinally. These materials was introduced by Lake's [1], and also they are special and fascinating materials for many industrial applications as follows; military applications $\rightarrow$ helmet, bullet proof vest, knee pad, gloce, protective wear, aircraft applications $\longrightarrow$ vanes for gas turbine engines, thermal protection, aircraft nose cones, wing panel, sounds and vibrations absorber rivet, automotive applications $\longrightarrow$ bumper, cushion,

*Corresponding author: Address: Faculty of Technology, Department of Mechanical and Manufacturing Engineering, Isparta Applied Sciences University, 32200, Isparta, TURKEY. E-mail address: berkayergene@sdu.edu.tr, Phone: +905542814943 
thermal protection, fastener, medical applications $\longrightarrow$ bandage, wound pressure pad, dental floss, artificial blood vessel, artificial skin, drug release unit, ligament anchors, surgical implants, oesophageal stents. One example of important applications of auxetic structures in aerospace and automotive engineering or in civil engineering is the absorption of powerful impacts such as explosive waves, so they are often used as the outer layer, safeguarding structures inside [2]. Attempts to increase damping capacity of mechanical structure or to decrease vibrations of mechanical systems used in these sectors have progressively increased, therefore, the cellular materials have received special attention of many researcher in this field [2-10]. Forexample, Assidi et al. [3] presented the composites with auxetic inclusions showing both an auxetic behavior and enhancement of their mechanical properties. Wan et al. [4] investigated the study of negative Poisson's ratios in auxetic honeycombs based on a large deflection model. Prawota shows the relationship between poisson ratio and ratio $(\mathrm{G} / \mathrm{K})$ of shear modulus $(\mathrm{G})$ with bulk modulus $(\mathrm{K})$ of auxetic and conventional material in Fig.1. He clarified that this structure gains the higher shear stiffness, strength, damping capacity, indentation resistance and fracture toughness [5].

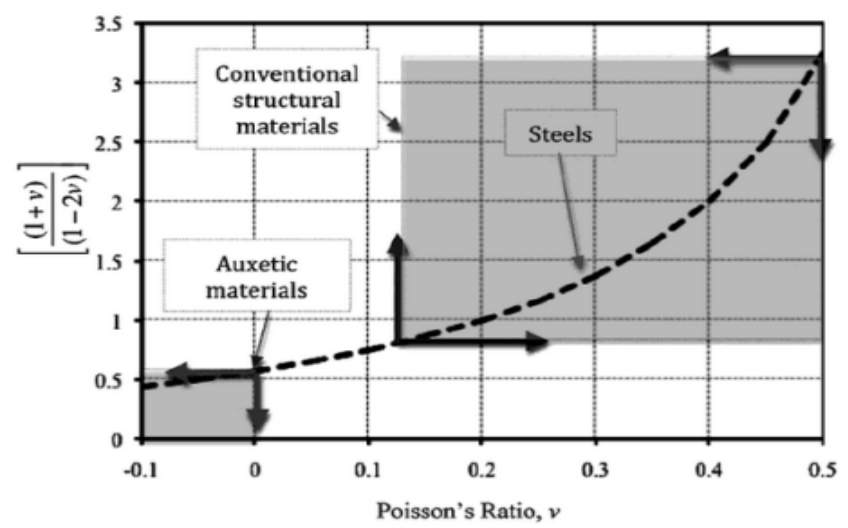

Figure 1. The relation between the poisson's ratio and the value of y axis for conventional and auxetic materials [5].

Damping may be represents by various parameters such as specific damping capacity, loss factor, Q factor and damping ratio. Damping capacity of them is known ability to dissipate elastic strain energy during mechanical vibrations. Cellular structures which especially showing the auxetic behaviour are superior in many practical applications in decreasing the propagation of vibrations efficiently [6,7], exhibiting enhanced indentation resistance [8].

Due to desire about manufacturing high strength structures at low density and high geometric freedom of additive manufacturing processes such as selective laser melting (SLM, [11-14]), fused deposition modelling (FDM, [15-17]), electron beam melting (EBM, [18;19]) very complex cellular structures can now be built. Also, Novak [20] indicates that additive manufacturing is a promising method at producing of cheaper, more complex and bigger auxetic structures. 


\section{Materials and Method}

Firstly, regular honeycomb structure with height $(\mathrm{h})=$ length (l) of $4,83 \mathrm{~mm}$, thickness (t) of $1.3 \mathrm{~mm}, \theta=30^{\circ}$ (Fig. 2-a) and reentrant structure with $\mathrm{h}=10 \mathrm{~mm}, \mathrm{l}=5 \mathrm{~mm}, \mathrm{t}=1 \mathrm{~mm}, \theta=30^{\circ}$ (Fig. 2-b) were designed to have same relative density in both structure type and to carry out the finite element analysis. The designed structures (Fig. 2) were exported to finite element program and then heat treated Ti-6Al-4V material at $800^{\circ} \mathrm{C}$ for 2 hours [21] after additive manufactured by DMLS (Direct Metal Laser Sintering) was chosen and its material constants; elasticity modulus of $120000 \mathrm{MPa}$, Poisson's ratio of 0.35 and density of $44.10^{-7} \mathrm{~N} / \mathrm{mm}^{3}$ were entered to the FEA program before determining element type and mesh properties of these structures for modal and structural analysis. In modal analysis, regular honeycomb and reentrant structures were used which can be seen in Fig. 2-a and Fig. 2-b. Furthermore, in this part of study, 8node185 and 4node182 as element types, thickness of $1.3 \mathrm{~mm}$, and element size of $0.65 \mathrm{~mm}$ were decided to use in FEA for getting homogenous mesh. After that, regular honeycomb and reentrant structures were fixed as encastre from the left side as boundary condition. Moreover, 10 modes was conducted using Block lanczos mode as mode-extraction method and vibration analysis were performed to obtain natural frequency values and to observe vibrational behaviour of these cellular structures. On the other hand, besides homogenous mesh, quad Quad4node42 element type with plane thickness option, thickness of $1.3 \mathrm{~mm}$ and element size of $0.65 \mathrm{~mm}$ was used because of carrying out 2D structural analysis of the structures which can be seen in Fig. 2-c and Fig. 2-d. Also, the left side of these structures were fixed as encastre like same in vibrational analysis procedure and $0.4 \mathrm{~mm}$ displacement along $-\mathrm{x}$ axis direction was applied to the right side of these structures as distinct from vibrational analysis in order to determine the mechanical behaviour such as stress and displacement which occurs on these unique structures under compression status. In addition, for both type of analysis (modal and structural analysis), material constants were used same like mentioned earlier above.

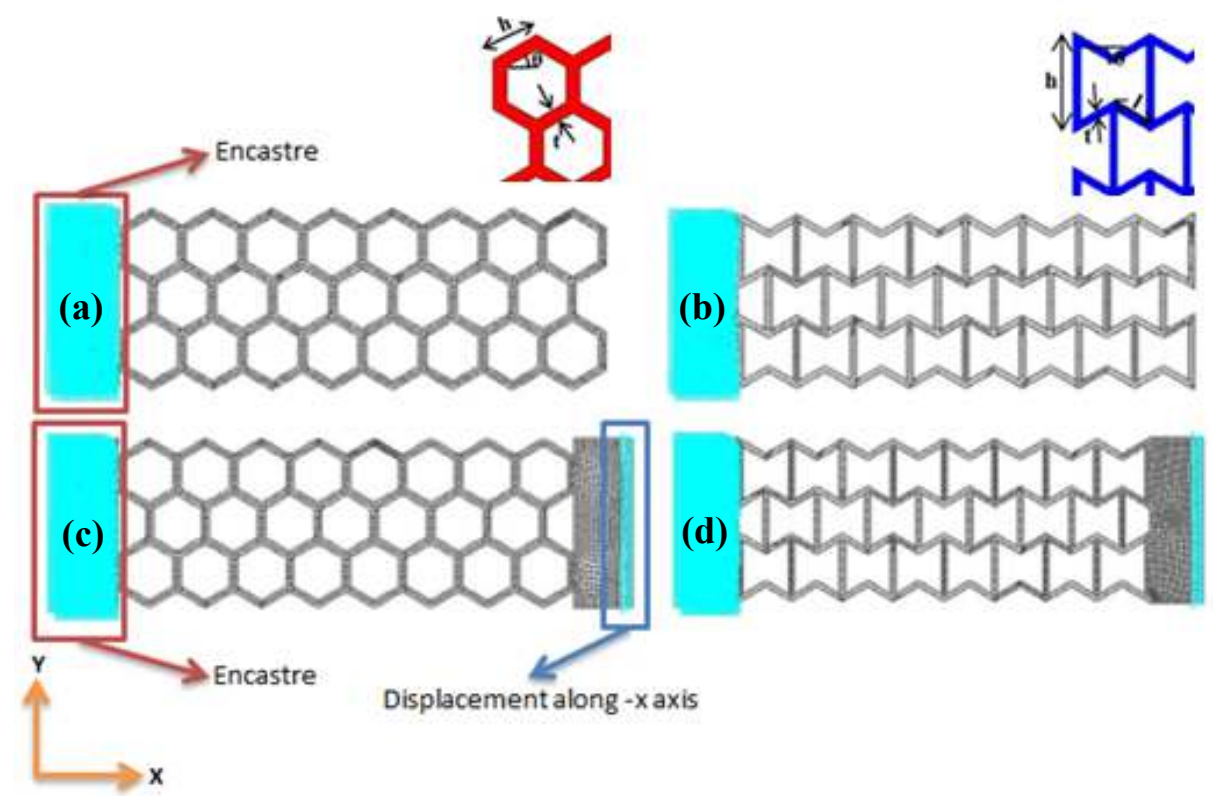

Figure 2. (a) Honeycomb structure for modal analysis (b) Reentrant structure for modal analysis (c) Honeycomb structure for structural analysis (d) Reentrant structure for structural analysis. 


\section{Results}

\subsection{Modal analysis results}

Fig.3-a and Fig. 3-b present frequency values for 10 modes and displacement vector sum of these structures at these frequencies respectively for honeycomb and reentrant structures. The obtained results from finite element analysis show that frequency values are always more in honeycomb structure than reentrant structure for all modes. Furthermore, frequency values increase to $197,230 \mathrm{~Hz}$ from $5,833 \mathrm{~Hz}$ for honeycomb and from $5,003 \mathrm{~Hz}$ to $151,34 \mathrm{~Hz}$ for reentrant during initial mode (mode I) to final mode (mode X). Besides, maximum displacement vector sum of $45,228 \mathrm{~mm}$ in 8 th mode for honeycomb and 49,954mm in 5th mode for reentrant structures. Also, the minimum displacement vector sum was observed in 9 th mode for honeycomb structure as $18,706 \mathrm{~mm}$ and in 10 th mode for reentrant structure as $20,051 \mathrm{~mm}$.
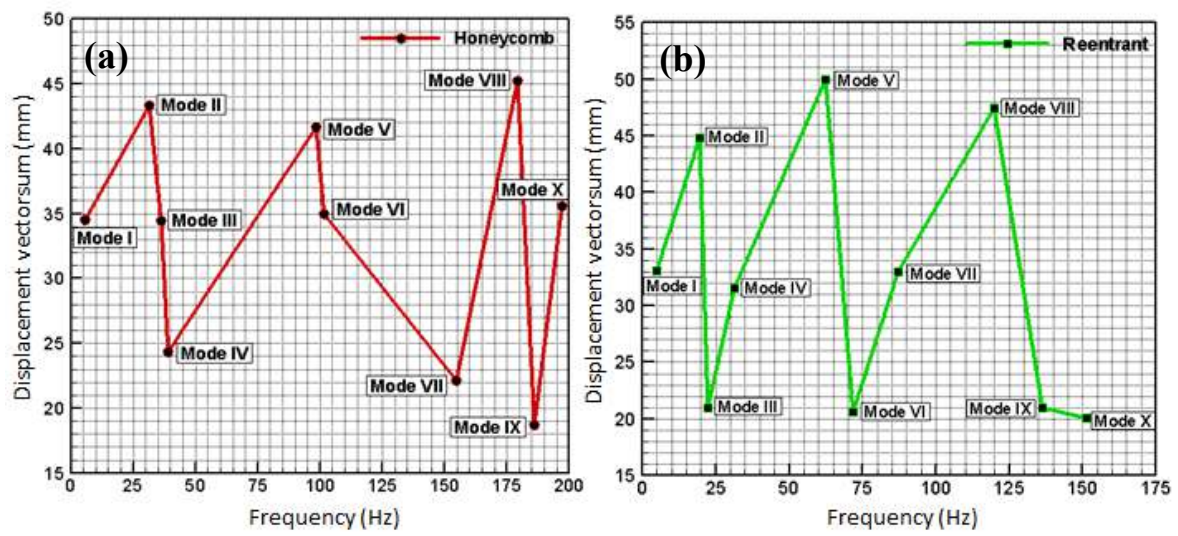

Figure 3. Displacement vectorsum vs frequency values (a) for honeycomb structure, (b) for reentrant structure.

In the light of finite element analysis carried out to understand the vibrational behaviour of honeycomb and reentrant structures, displacement along y axes $\left(\Delta \mathrm{l}_{\mathrm{y}}\right)$ varies between $-2.898 \mathrm{~mm}$ in 2nd mode to $24.028 \mathrm{~mm}$ in 4 th mode for honeycomb structure while $\Delta \mathrm{l}_{\mathrm{y}}$ of reentrant structure changes between $-6.003 \mathrm{~mm}$ in 10 th mode and $20.793 \mathrm{~mm}$ in $3 \mathrm{rd}$ mode. Besides, it can be evaluated that without 6 th and 7 th mode these structures, they exhibit similar trend in case of having $\Delta \mathrm{l}_{\mathrm{y}}$ along positive y axis or negative y axis (Fig. 4).
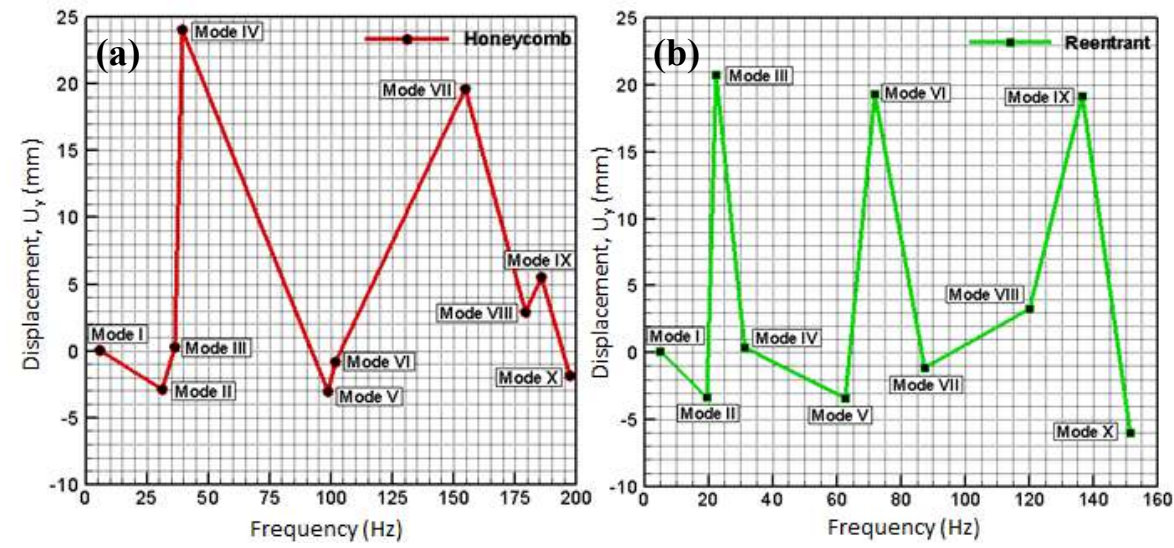

Figure 4. Displacement $\left(\mathrm{U}_{\mathrm{y}}\right)$ vs frequency values (a) for honeycomb structure, (b) for reentrant structure 
Besides obtaining displacement vector sum and displacement along y axis of these cellular structures, natural frequencies and appearance of these structures were introduced obviously in Table 1.

Table 1. Natural Frequency values of honeycomb and reentrant structures and their appearance at related modes.

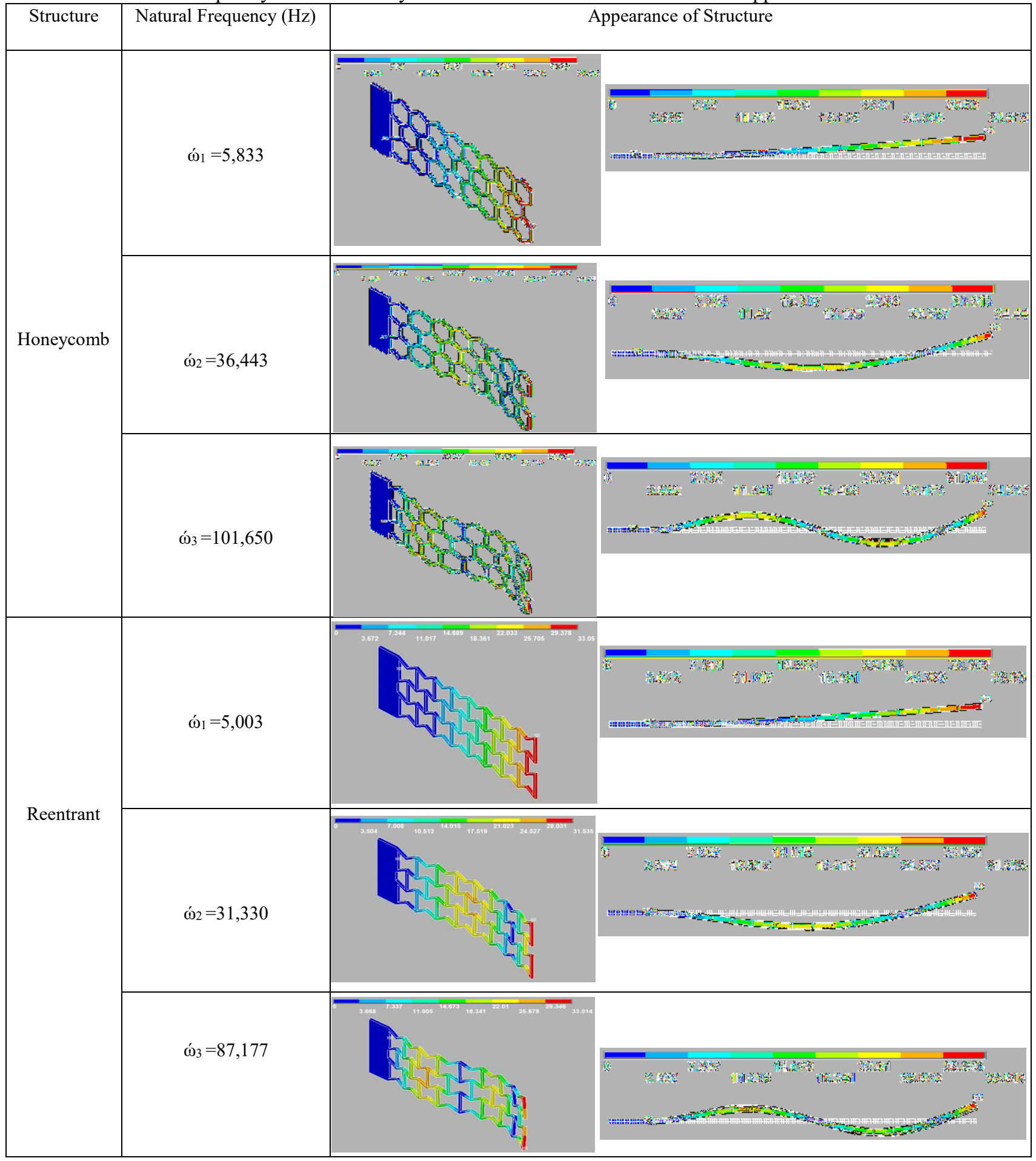


According to Table 1, natural frequencies $\left(\hat{\omega}_{1}, \omega_{2}, \omega_{3}\right)$ were obtained as $5,833 \mathrm{~Hz}, 36,443 \mathrm{~Hz}$ and $101,650 \mathrm{~Hz}$ for honeycomb structure and $5.003 \mathrm{~Hz}, 31,330 \mathrm{~Hz}$ and $87,177 \mathrm{~Hz}$ for reentrant structure respectively. Moreover, natural frequency values for honeycomb are higher than reentrant structure evidently as can be seen in Table 1. Besides, displacement vector sum values of honeycomb structure seem more than values of reentrant structure for every mode which were used to determine natural frequencies of these cellular structures.

\subsection{Auxetic behaviour and stress results}

In this part of study, as mentioned earlier left side of the structures were fixed as encastre and 0.4 $\mathrm{mm}$ displacement was applied to the right side of the structures along $-\mathrm{x}$ axes to present the auxetic behaviour of reentrant structure and get normal stresses $\left(\sigma_{\mathrm{x}}\right.$ and $\left.\sigma_{\mathrm{y}}\right)$, von-mises stress $\left(\sigma_{\mathrm{vm}}\right)$ and shear stress $\left(\tau_{\mathrm{xy}}\right)$ which occur on the structures after $0.4 \mathrm{~mm}$ displacement enforced. In the literature, many researchers have been interested in auxetic materials in viewpoint of material development [22-24] and mechanical modelling [25]. In this research, auxetic behaviour of reentrant structure was obtained as compatible to earlier studies has been done in literature and the difference in movement of honeycomb and reentrant structure can easily be seen in Fig. 5-b. Also, black points on model show the initial position for both structures and red and green points indicate the final position of honeycomb and reentrant structure respectively. By interpreting the finite element results, it is understood that reentrant structure exhibits shrinkage along y axis although honeycomb structure expands along same axis. That's the point; reentrant structures differ from honeycomb structure because of having negative Poisson's ratio as an effect of behaving auxeticly. According to Fig. 5-a, honeycomb structure has $0.164 \mathrm{~mm}$ expansion along y axis and reentrant structure has $0.179 \mathrm{~mm}$ shrinkage along y axis.
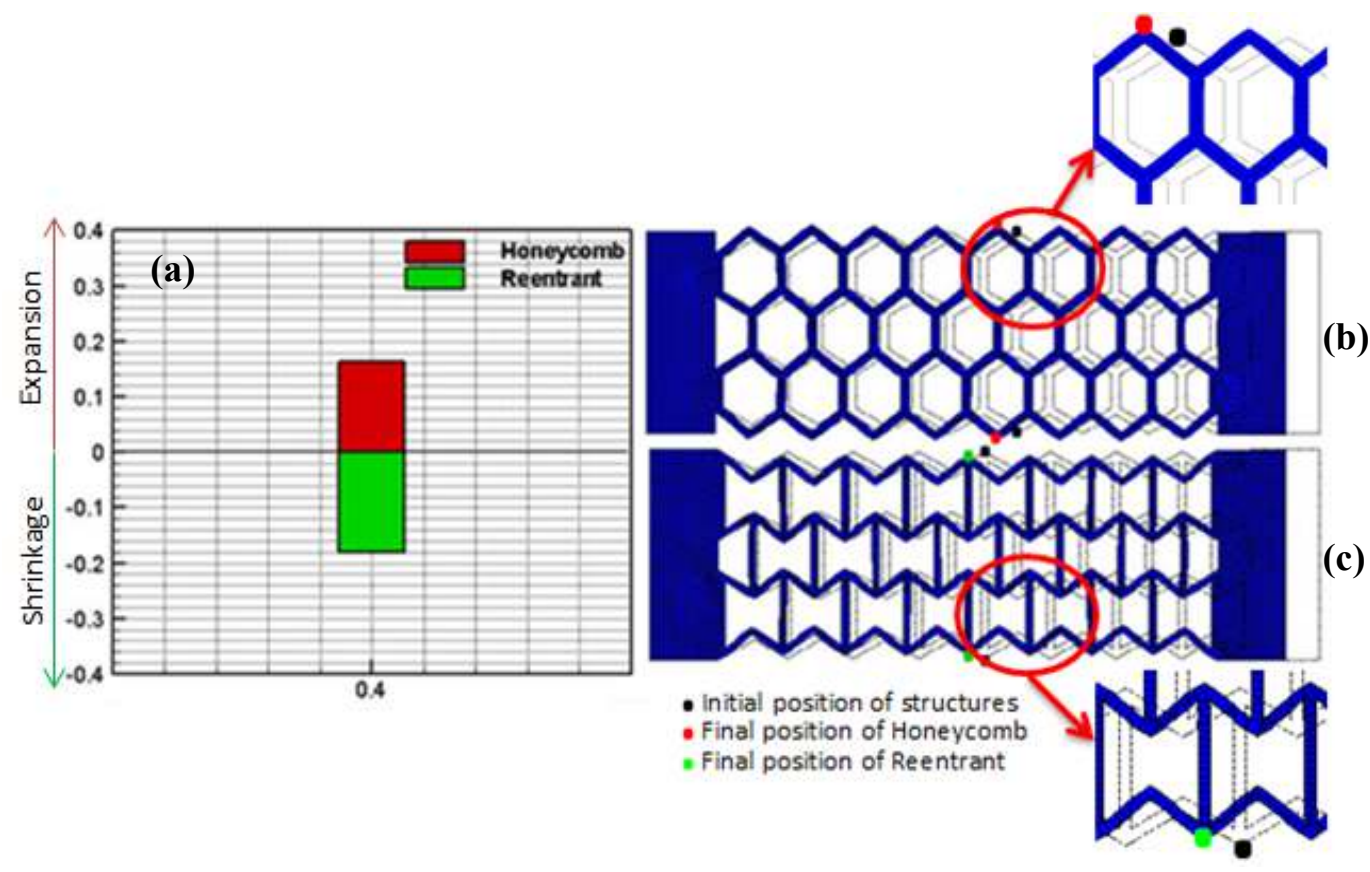

Figure 5. (a) Expansion or shrinkage behavior of honeycomb and reentrant structures, (b) view of honeycomb structure after $0.4 \mathrm{~mm}$ displacement applied, (c) view of reentrant structure after $0.4 \mathrm{~mm}$ displacement applied. 
In Figure 6, it is worth noticing that all stress results $\left(\sigma_{\mathrm{x}}, \sigma_{\mathrm{y}}, \sigma_{\mathrm{vm}}\right.$, and $\left.\tau_{\mathrm{xy}}\right)$ have lower values in reentrant structure than honeycomb structure. Besides, $\sigma_{\mathrm{x}}$ of $918.269 \mathrm{MPa}$ in honeycomb structure and $\sigma_{\mathrm{x}}$ of $692.94 \mathrm{MPa}$ in reentrant structure were obtained. Also, $\sigma_{\mathrm{y}}$ of $364.452 \mathrm{MPa}$ and $243.156 \mathrm{MPa}$ occurred on honeycomb and reentrant structure respectively. Furthermore, it is an important point that honeycomb structure keeps within $\sigma_{\mathrm{vm}}$ of $840.386 \mathrm{MPa}$ and this value is $676.093 \mathrm{MPa}$ for reentrant structure although both of the cellular structures have same displacement value $0.4 \mathrm{~mm}$ along $\mathrm{x}$ axis. In contrast to other stress, $\tau_{\mathrm{xy}}$ values are close to each other and $\tau_{\mathrm{xy}}$ value of honeycomb was $269,912 \mathrm{MPa}$ and $\tau_{\mathrm{xy}}$ value of reentrant was $261.188 \mathrm{MPa}$.

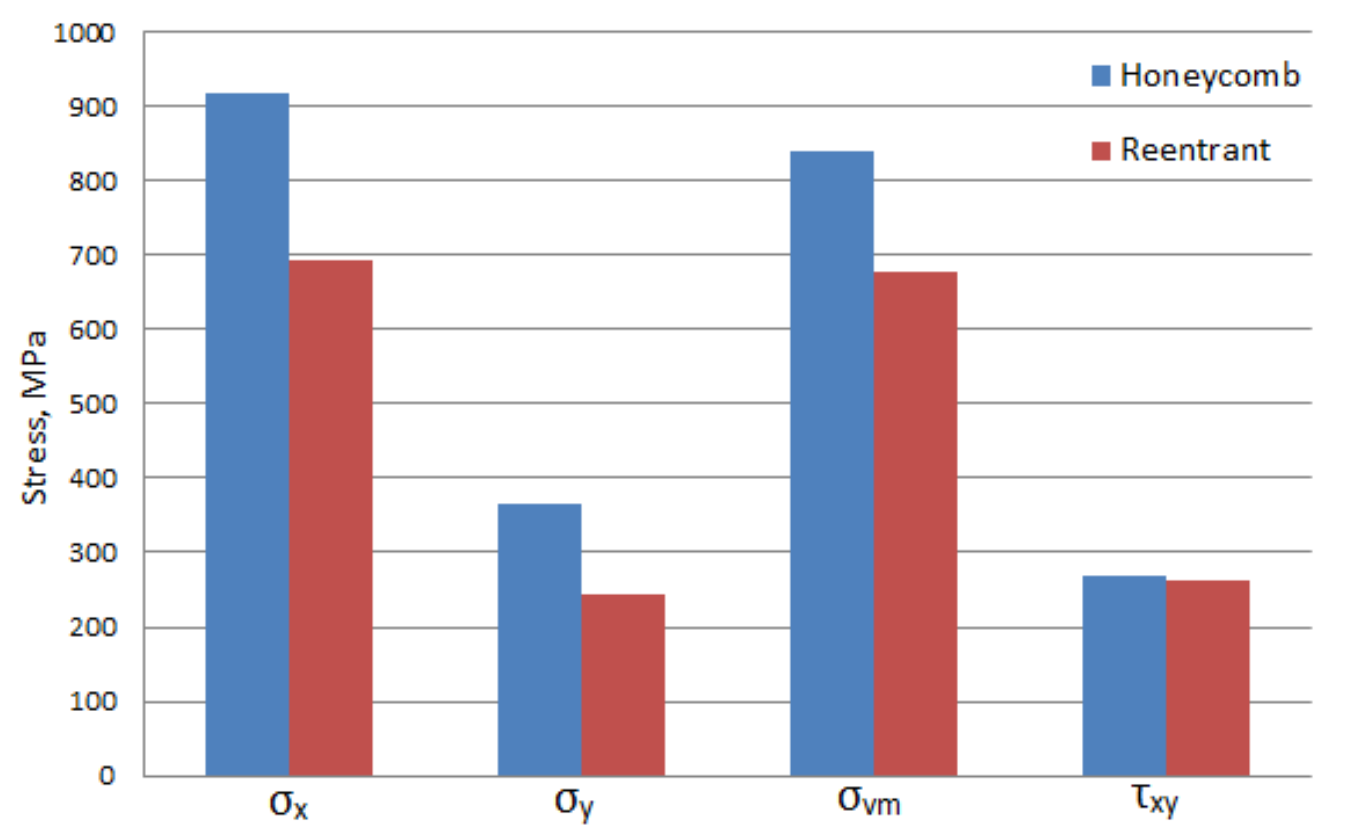

Figure 6. Stresses occurred on honeycomb and re-entrant structure under defined boundary conditions

On the other hand, at the same displacement $0.4 \mathrm{~mm}$ along $\mathrm{x}$ axis was enforced for honeycomb and reentrant structures, less $\sigma_{\mathrm{x}}, \sigma_{\mathrm{y}}, \sigma_{\mathrm{vm}}$, and $\tau_{\mathrm{xy}}$ occurred on reentrant structure and more displacement along y axis was observed in reentrant structure in comparison with honeycomb structure. These obtained stress and displacement results from finite element analysis express that using of reentrant structure can be advantageous about energy absorption instead of using honeycomb structure in some areas like energy absorption takes an important role. Also, Yang [26] reported that negative Poisson's ratio material has good ability of energy absorption according to numerical results in its study about structural response of a negative Poisson's ratio structure with regular reentrant cell shape under the rigid wall crushing. Furthermore, Dong [27] mentions about better ability to absorb impact energy as well as increase structural strength makes NPR structures outperform the conventional structures in the various engineering applications when integrated with 3D printing technology. 


\section{Conclusions}

In the light of this survey, the following results can be expressed;

- The obtained results from finite element analysis show that frequency values are always more in honeycomb structure than reentrant structure for all modes.

- Furthermore, frequency values increase to $197,230 \mathrm{~Hz}$ from $5,833 \mathrm{~Hz}$ for honeycomb and from $5,003 \mathrm{~Hz}$ to $151,34 \mathrm{~Hz}$ for reentrant during initial mode (mode I) to final mode (mode $\mathrm{X})$.

- Maximum displacement vector sum of $45,228 \mathrm{~mm}$ in 8 th mode for honeycomb and $49,954 \mathrm{~mm}$ in 5 th mode for reentrant structures. Also, the minimum displacement vector sum was observed in 9th mode for honeycomb structure as $18,706 \mathrm{~mm}$ and in 10 th mode for reentrant structure as $20,051 \mathrm{~mm}$.

- In the light of finite element analysis carried out to understand the vibrational behaviour of honeycomb and reentrant structures, displacement along y axes $(\Delta$ ly) varies between $2.898 \mathrm{~mm}$ in $2 \mathrm{nd}$ mode to $24.028 \mathrm{~mm}$ in 4 th mode for honeycomb structure while $\Delta$ ly of reentrant structure changes between $-6.003 \mathrm{~mm}$ in 10 th mode and $20.793 \mathrm{~mm}$ in $3 \mathrm{rd}$ mode.

- According to Table 1, natural frequencies $\left(\dot{\omega}_{1}, \dot{\omega}_{2}, \omega_{3}\right)$ were obtained as $5,833 \mathrm{~Hz}$, $36,443 \mathrm{~Hz}$ and $101,650 \mathrm{~Hz}$ for honeycomb structure and $5.003 \mathrm{~Hz}, 31,330 \mathrm{~Hz}$ and $87,177 \mathrm{~Hz}$ for reentrant structure respectively. Moreover, natural frequency values for honeycomb are higher than reentrant structure evidently as can be seen in Table 1.

- Non-auxetic behaviour of honeycomb structure and and auxetic behaviour of reentrant structure were observed (Fig. 5)

- That's the point; reentrant structures differ from honeycomb structure because of having negative Poisson's ratio as an effect of behaving auxeticly. According to Fig. 5-a, honeycomb structure has $0.164 \mathrm{~mm}$ expansion along y axis and reentrant structure has $0.179 \mathrm{~mm}$ shrinkage along y axis.

\section{References}

[1] R. Lakes, Foam structures with a negative Poisson's ratios. Science 235, (1987) 1038-1040.

[2] N. DinhDuc, K. Eock, N. DucTuan, P. Tran, N. DinhKhoa,. New approach to study nonlinear dynamic response and vibration of sandwich composite cylindrical panels with auxetic honeycomb core layer. Aerospace Science and Technology, 2017, 70, 396-404

[3] M. Assidi, J. Ganghoffer. Composites with auxetic inclusions showing both an auxetic behavior and enhancement of their mechanical properties. Compos. Struct, 2012, 94, 2373-2382

[4] H. Wan, H. Ohtaki, S. Kotosaka, et al., A study of negative Poisson's ratios in auxetic honeycombs based on a large deflection model. Eur. J. Mech., 2014, 23, 95-106. 
[5] Y. Prawota, Solid Mechanics for Materials Engineers: Auxetic materials seen from the mechanics point of view, Chapter 15, USA, 2013.

[6] F. Scarpa and G. Tomlinson. Theoretical characteristics of the vibration of sandwich plates with in-plane negative Poisson's ratio values. Journal of Sound and Vibration, 2000, vol. 230(1).

[7] X.W. Zhang and D.Q. Yang. A novel marine impact resistance and vibration isolation cellular base. Journal of Vibration and Shock, 2015, 34(10), 40-45.

[8] R. S. Lakes and K. Elms. Indentability of conventional and negative Poisson's ratio foams. Journal of Composite Materials, 1993, 27(12), 1193-1202.

[9] D. Mousanezhad, H. Ebrahimi, B. Haghpanah, R. Ghosh, A. Ajdari, A.M.S. Hamouda, A. Vaziri. Spiderweb honeycombs. International Journal of Solids and Structures, 2015, 66, 218227.

[10] C. Yang, H. Vora and Y. Chang. Behavior of auxetic structures under compression and impact forces. Smart Mater. Struct., 2018, 27(2), 1-12.

[11] B. Gorny, T. Niendorf, J. Lackmann, M. Thoene, T. Troester and H. J. Maier. In situ characterization of the deformation and failure behavior of non-stochastic porous structures processed by selective laser melting. Mater. Sci. Eng. A, 2011, 528(27), 7962-7967.

[12] V. Weißmann, J. Wieding, H. Hansmann, N. Laufer, A. Wolf and R. Bader. Specific yielding of selective laser-melted Ti6A14v open-porous scaffolds as a function of unit cell design and dimensions. Metals, 2016, 6(7), 1-19.

[13] S. Li, H. Hassanin, M.M. Attallah, N.J. Adkins, K. Essa. The development of TiNi-based negative Poisson's ratio structure using selective laser melting. Acta Mater., 2016, 105, 75-83.

[14] O. Rehme and C. Emmelmann. Selective laser melting of honeycombs with negative poisson's ratio. JLMN-Journal of Laser Micro/Nanoengineering, 2009, 4(2), 128-134.

[15] P. Dziewit, P. Platek, J. Janiszewski, M. Sarzynski, M. Grazka, R. Paszkowski. Mechanical response of additive manufactured regular cellular structures in quasi-static loading conditionsPart I experimental investigations. Proceedings of the 7th International Conference on Mechanics and Materials in Design, 2017, 1061-1074.

[16] A. Ingrole, A. Hao, R. Liang. Design and modeling of auxetic and hybrid honeycomb structures for in-plane property enhancement. Materials and Design, 2017, 117, 72-83.

[17] B. Pandaa, M. Leitea, B. B. Biswalb, X. Niuc, A. Gargc. Experimental and numerical modelling of mechanical properties of 3D printed honeycomb structures. Measurement,2018, 116, 495-506. 
[18] L. Yang, O. Harrysson, H. West, D. Cornier. Mechanical properties of 3D re-entrant honeycomb auxetic structures realized via additive manufacturing. International Journal of Solids and Structures, 2015, 69-70, 475-490.

[19] L. Yang, O. Harrysson, H. West, D. Cornier. Compressive properties of Ti-6Al-4V auxetic mesh structures made by electron beam melting. Acta Materialia, 2012, 60, 3370-3379.

[20] N. Novak, N. Vesenjak, M. Ren, Z. Nejc, M. Vesenjak, Z. Ren. Auxetic Cellular Materials a Review. Journal of Mechanical Engineering, 2016, 62(9), 485-493.

[21] SLM/DMLS Titanium Ti6A14V, Crp Meccanica, https://www.crpmeccanica.com/PDF/SLM-DMLS_TitaniumTi6Al4v_CRP.pdf, Last access: 15.05.2018

[22] K.E. Evans, K.L. Alderson. Auxetic materials: the positive side of being negative. Eng. Sci. Edu. J., 2000, 9, 148-154.

[23] E.A. Friis, R.S. Lakes, J.B. Park. Negative Poisson's ratio polymeric and metallic materials. J. Mater. Sci., 1988, 4406-4414.

[24] G.N. Greaves, A.L. Greer, R.S. Lakes, T. Rouxel. Poisson's ratio and modern materials. Nature Materials, 2011, 10, 823-837.

[25] J.B. Choi, R.S. Lakes. Fracture toughness of re-entrant foam materials with a negative Poisson's ratio: experiment and analysis. Int. J. Fracture., 1996, 80, 73-83.

[26] S. Yang, C. Qi, D. Guo, D. Wang. Energy absorption of an re-entrant honeycombs with negative poisson's ratio. Applied Mechanics and Materials, 2012, 148-149, 992-995.

[27] G. Dong, L. Yang, Y. Gao, D. Liu. An efficient energy absorbing structure inspired by energy harvesting device. International Conference on Modeling, Simulation and Optimization (MSO 2018), 2018, 450-455. 